\title{
A Molecular Dynamic Study of Charged Nanofilm Interaction with Negative Lipid Bilayer
}

\author{
Alexey A. Tsukanov ${ }^{1, a)}$ and Sergey G. Psakhie ${ }^{1,2, b)}$ \\ ${ }^{1}$ Skolkovo Institute of Science and Technology, Skolkovo, 143025, Russia \\ ${ }^{2}$ National Research Tomsk Polytechnic University, Tomsk, 634050, Russia \\ a) Corresponding author: a.tsukanov@skolkovotech.ru \\ ${ }^{\text {b) }}$ sp@ispms.tsc.ru
}

\begin{abstract}
Hydrophobic and functionalized nanoparticles of different sizes and shapes have a various biomedical application, in particular anticancer therapy. It is known that charged nanoparticles may bind lipids and membrane proteins as well as cause lipid bilayer disruption. We have performed preliminary molecular dynamic simulations to investigate the effect of positively charged synthetic nanofilm, imitating a fragment of the two-dimensional folded $\mathrm{AlOOH}$ structure, on the POPE/POPG lipid membrane. It has been shown that the synthetic nanofilm with frozen coordinates tightens the membrane and binds lipid headgroups. Furthermore, the presence of the positively charged nanofilm perturbs the cation concentration in the near-surface membrane region.
\end{abstract}

Keywords: lipid membrane, charged nanofilm, cancer, antitumor therapeutics, molecular dynamics, ion transport, aluminum oxyhydroxide

\section{INTRODUCTION}

Recently, much attention is given to the studies of interaction of different nano-objects with lipid membranes and their derivatives. The interest is primarily due to a wide range of biomedical applications such as drugs, therapeutics and genes delivery, as well as inhibition and deactivation functioning of membrane proteins. The promising direction in this case is the using of nanoparticles having a surface charge [1-3]. These nanoparticles penetrate through the plasma membrane into the cell, where they interact with organelles membranes, in particular, mitochondria membranes, causing their destruction. The electrostatic effect on the outer part of the membrane is another way to influence the life activity of cancer cells [4]. It is known that the so-called agglomerates of lowdimensional (2D) folded sheet structures have a positive surface charge [5-7].

\section{EFFECT OF CHARGED NANO-OBJECTS ON LIPID MEMBRANES}

Interaction of charged nano-objects with lipid membranes is the subject of numerous experimental and theoretical studies. Computer simulations, especially molecular dynamics methods, have an important place among the theoretical methods of studying such nanoscale processes. In this aspect, main attention is given to several groups of charged nano-objects. Here, we may formally identify 5 groups: (a) small polar/charged molecules and ions; (b) charged peptides, proteins and drugs; (c) functionalized fullerenes, dendrimers, and nanoparticles with inorganic core; (d) one-dimensional nano-objects (functionalized carbon nanotubes, rods, nanowires); (e) twodimensional nano-objects (functionalized graphene nanosheets, inorganic nanofilms and substrate).

The shape, size and charge of the nano-object determine the manner of its interaction with the cell membrane [810]. As has been shown, the neutral and hydrophobic nanoparticles and their aggregates may penetrate the lipid membrane by passive diffusion $[11,12]$. Not too large charged (or polar) nanoparticles prefer to be adsorbed in the lipid head group region [13-15]. Large charged proteins and dendrimers (with a large generation number) bind to

International Conference on Physical Mesomechanics of Multilevel Systems 2014

AIP Conf. Proc. 1623, 639-642 (2014); doi: 10.1063/1.4899026

(C) 2014 AIP Publishing LLC $978-0-7354-1260-6 / \$ 30.00$ 


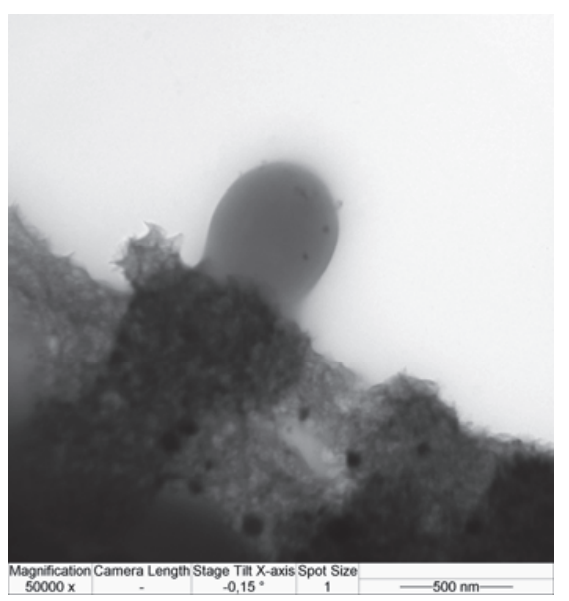

FIGURE 1. The "caught" staphylococcus aureus

the cell membrane, causing the bilayer deformation [16], the pore or discontinuity formation [17] in the case of the elastic tension of the membrane [18]. One-dimensional nano-objects such as hydrophobic carbon nanotubes, bundles of nanotubes, asbestos fibers, gold nanowires, etc can penetrate through the cell membrane by puncturing or endosome formation and receptor-mediated mechanism $[19,20]$. This allows us to consider the single-wall nanotubes and fullerenes as nanocarriers for anticancer drugs, such as paclitaxelum (PTX) [21]. Behavior of functionalized nanotubes, as well as wrapped ones, was studied by molecular dynamics in [22-24]. As has been shown, such nano-objects in certain cases may penetrate into the lipid bilayer and distort it. Not only the magnitude but also the sign of the nanoparticle surface charge influences the mechanism of its impact on the cell membrane. For example, in [25] the interaction of hydrophobic and functionalized gold nanoparticles with neutral or negatively charged membranes was studied by molecular dynamics simulations. It has been shown that anionic nanoparticle prefers to avoid any interaction with the negatively charged membrane, but it binds to the hydrophilic surface region of the neutral membrane. Cationic nanoparticle with low surface charge density binds to the surface of both types of membranes. In case of higher surface density of positive charge, the nanoparticle strongly interacts with the membrane, being immersed into the bilayer. [25]. Thus a single-layer embedded micelle or an embedded bilayer vesicle may be formed around positively charged nanoparticles [26]. Such a vesicle, containing a nanoparticle, may break away from the membrane, forming endosome. It is noted that there is a range of values for the charge density, in which the efficient endocytosis occurs and no destruction of the membrane is observed [25]. Further increase of charge density may result in cell membrane disruption.

The interaction of positively charged low dimensional nano-objects with cell membranes, particularly with the membrane of staphylococcus aureus bacteria, is observed experimentally. Figure 1 demonstrates a staphylococcus aureus bacterium caught by the positively-charged low-dimensional structures of Al oxyhydroxide. The last one was synthesized using the technology described in [27].

We also expect that the aluminum oxyhydroxide nanofilm binds to extracellular polar groups of membrane proteins. Such an action, for example, may inhibit the ion channels functioning of the bonded/caught cancer cell. Furthermore, the presence of a positively charged nano-film near the cell membrane may perturb ion concentration, reducing the number of cations beside the outer surface of the membrane, thereby it alters the electrochemical gradient.

To test our assumptions, we conducted simple full-atom molecular dynamics simulation. In this short report, we discuss the first stage of the numerical simulation: interaction of negatively charged cell membranes (composed of two types of lipids) with a fragment of the two-dimensional folded sheet structure of aluminum oxyhydroxide, for which a simple model was used.

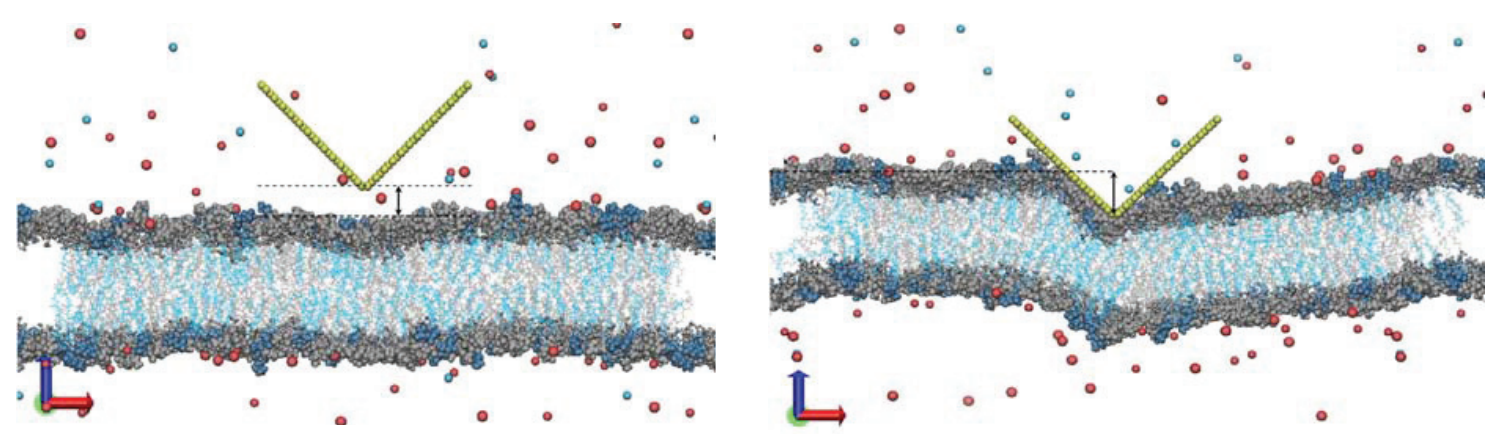

FIGURE 2. Snapshots of the system at 0 (left) and 90 ns (right). Color code: gray lipids—POPE, blue lipids—POPG, blue ions $-\mathrm{Cl}^{-}$, red big ions $-\mathrm{Na}^{+}$, red small ions $-\mathrm{K}^{+}$, yellow-nanofilm, water omitted for clarity. Image was created with VMD [32] 


\section{MODEL}

A fragment of the two-dimensional folded $\mathrm{AlOOH}$ structure was modeled as infinite along $y$-axis sheet (Fig. 2(a)), which was bent at an angle of $90^{\circ}$ and its vertex was oriented towards the membrane. The surface charge density of the structure was $+0.1 \mathrm{cl} / \mathrm{m}^{2}$. The periodic boundary conditions in all directions were used. Nanofilm coordinates were fixed during the whole simulation period. The lower edge of the nanofilm was placed to $z=36 \AA$ (about $10 \AA$ above the membrane surface), the plane $z=0$ was in the middle of the bilayer. The membrane was composed of two lipid typesneutral POPE and anionic POPG phospholipids with charge $-1 e, \mathrm{PG}: \mathrm{PE}$ ratio was about $\sim 0.3$.

Simulation protocol: All-atom molecular dynamics simulation was performed using the LAMMPS molecular dynamics package (Sandia National Laboratory) [28] and CHARMM27 force field [29]. The lipid membrane was composed of 230 neutral POPE and 70 anionic POPG phospholipids. The total membrane charge was $-70 e$. The membrane was preliminarily equilibrated in 55000 TIP3P water molecules with $35 \mathrm{~K}^{+}$and $35 \mathrm{Na}^{+}$ions (neutral system). The simulation box dimensions were $200 \times 45 \times 220 \AA$. When synthetic nanofilm was added to the system, overlapped water molecules were removed. Moreover, 25 random water molecules were replaced by $25 \mathrm{Cl}^{-}$anions to compensate the nanofilm positive charge. The total number of atoms was about 200000 . The synthetic nanofilm, mimicking a fragment of AlOOH twodimensional nanomaterial, was composed of 500 abstract A atoms with frozen coordinates.

The Lennard-Jones parameters for A were chosen equivalent to the corresponding values of a neutral aluminum atom $\varepsilon=-4.02 \mathrm{kcal} / \mathrm{mole}, \sigma=1.4625 \AA$ [30]. The partial charge of A atoms was set to $0.05 e$ to provide a uniform surface charge density of the nanofilm $+0.1 \mathrm{cl} / \mathrm{m}^{2}$ (total nanofilm charge was $+25 e$ ). A cutoff distance of the Van der Waals potential was $10 \AA$. The LJ potential was smoothly shifted to zero between 8 and $10 \AA$. The cutoff of $20 \AA$ was used for pairwise Coulomb interaction. The full electrostatics was calculated using PPPM (particleparticle particle-mesh) algorithm with an accuracy of $10^{-4}$ [31]. Simulations were performed in the NPT ensemble. Temperature and pressure were maintained at $303 \mathrm{~K}$ and $1 \mathrm{~atm}$., respectively. The time step for simulations was $1 \mathrm{fs}$.

\section{RESULTS AND DISCUSSION}

The results of the molecular dynamics calculations showed that during the first 20-30 ns the membrane was pulled up by the nanofilm due to electrostatic attraction, travelling a distance of $10 \AA$. It should be noted that the membrane moves translationally like a "piston" because of the small size of the computational domain along the $x$ axis, $20 \mathrm{~nm}$. After the moment of contact, the membrane continues crawling over the nanofilm due to both electrostatic and van der Waals forces. At a simulation time of $90 \mathrm{~ns}$, the vertex of the nanofilm was immersed into the membrane at $15 \AA$ (Fig. 2(b)). A strong (non-covalent) bond between the membrane and the nanofilm was formed.
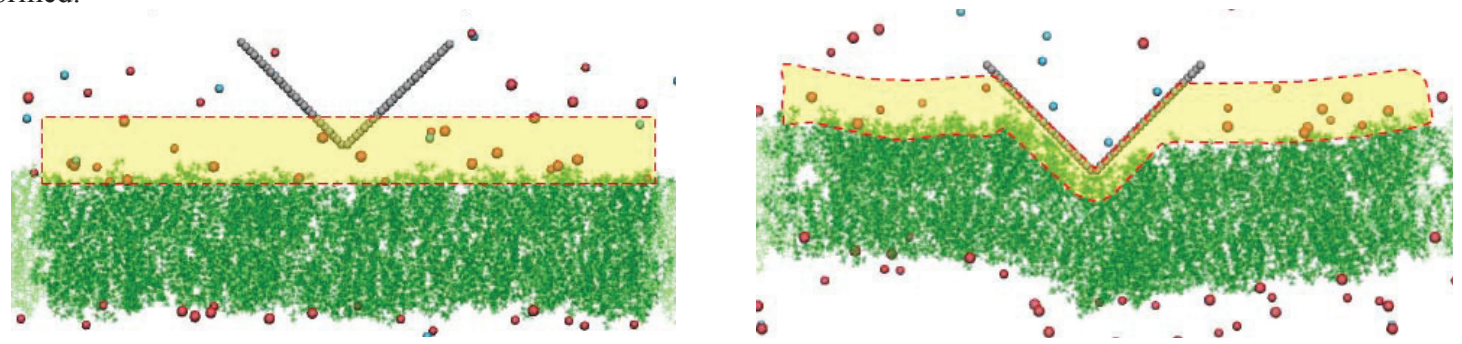

FIGURE 4. Left: $0 \mathrm{~ns}$, the cations concentrated near the surface (the membrane was previously equilibrated with cations), anions uniformly distributed in water. Right: $90 \mathrm{~ns}$, the concentration of cations was reduced by about 1.4 times within $20 \AA$ from the membrane (light yellow zones). Image was created with VMD 
In the contact zone the perturbation of the lipids head groups orientation was observed. Lipids prefer to orient the phosphate groups and oxygen atoms toward the nanofilm. The partial charges of oxygen are about $(-0.6 \div-0.8) e$. The positively charged amino groups of POPE lipids head groups were mostly turned away from the nanofilm (Fig. 3). We also noted that water molecules were "pressed" by the nanofilm from the contact region, leading to full dehydration in this zone. Moreover, a reduction in the concentration of $\mathrm{Na}^{+}$and $\mathrm{K}^{+}$ions was observed near the surface of the upper bilayer leaflet. For example, the amount of cations was reduced by about 1.4 times within $20 \AA$ from the membrane (Fig. 4).

\section{SUMMARY}

We have studied the interaction of the cell membrane with the positively charged AlOOH nanofilm using a simple model. The results of simulations indicate that the positively charged nanofilm attracts the membrane and binds with it. The concentration of cations is reduced near the membrane surface. Perturbations of the lipid head group structure and dehydration of the membrane surface are observed. We suppose that the interaction of aluminum oxyhydroxide with cell membranes would also affect the functioning of the ion channels.

We assume that the remarkable physical properties provide $\mathrm{AlOOH}$ nanostructures with great opportunities for biomedical applications such as anticancer therapies.

The reported study was supported by Grant 14-23-00096 of the Russian Science Foundation. The simulation protocol was developed under support of the Skolkovo Institute of Science and Technology. Calculations were performed under support of the Supercomputing Center of Lomonosov Moscow State University [33].

\section{REFERENCES}

1. W. Jiang, B. Y. S. Kim, J. T. Rutka, and W. C. W. Chan, Nat. Nanotech. 3, March (2008).

2. Weibo Cai, Ting Gao, Hao Hong, and Jiangtao Sun, Nanotechnology Sci. Applicat. 1, 17 (2008).

3. B. D. Chithrani, A. A. Ghazani, and W. C. W. Chan, Nano Lett. 6(4), D (2006).

4. R. R. Arvizo, O. R. Miranda, M. A. Thompson, et al., Nano Lett. 10(7), 2543 (2010).

5. B. Schneiderov, J. Demel, J. Plestil, P. Janda, J. Bohuslav, et al., J. Mater. Chem. A, 2013, 1, 11429-11437

6. Y. Kuang, L. Zhao, S. Zhang, F. Zhang, M. Dong, and S. Xu, Materials 3, 5220 (2010).

7. Q. Wu, A. Olafsen, Ø. B. Vistad, J. Roots, and P. Norby, J. Mater. Chem. 15(44), 4695 (2005).

8. V. V. Ginzburg, Nano Lett. 7, 3716 (2007).

9. K. Yang and Y. Q. Ma, Nat. Nanotech. 5, 579 (2010).

10. Shikha Nangia and Radhakrishna Sureshkumar, Langmuir 28(51), 17666 (2012).

11. J. Wong-Ekkabut, S. Baoukina, W. Triampo, I. M. Tang, et al., Nat. Nanotech. 3, 363 (2008).

12. S. Zhang, Y. Mu, J. Z. H. Zhang, and W. Xu, PLoS ONE 8(10), e77436 (2013).

13. J. N. Sachs and T. B. Woolf, J. Am. Chem. Soc. 125, 8742 (2003).

14. A. K. Sum, R. Faller, and J. J. de Pablo, Biophys. J. 85(5), 2830 (2003).

15. A. K. Sum and J. J. de Pablo, Biophys. J. 85(6), 3636 (2003).

16. O. V. Levtsova, I. D. Davletov, O. S. Sokolova, and K. V. Shaitan, Biofizika 56, 242 (2011).

17. H. Lee and R. G. Larson, J. Phys. Chem. B 112, 12279 (2008).

18. C. L. Ting and Z. G. Wang, Biophys. J. 100(5), 1288-97 (2011).

19. X. H. Shi, Y. Kong, and H. J. Gao, Acta Mech. Sin. 24, 161.

20. X. Shi, A. von dem Bussche, R. H. Hurt, A. B. Kane, and H. Gao, Nat. Nanotech. 6, 714.

21. S. Z. Mousavi, S. Amjad-Iranagh, Y. Nademi, and H. Modarress, J. Memb. Biol. (2013) doi:10.1007/s00232-013-9587-y.

22. S. Kraszewski, A. Bianco, M. Tarek, and C. Ramseyer, PLoS ONE 7(7), e40703 (2012).

23. S. Baoukina, L. Monticelli, and D. P. Tieleman, J. Phys. Chem. B 117, 12113-23 (2013).

24. H. Lee, J. Phys. Chem. B 117, 1337 (2013).

25. J. Lin, H. Zhang, Z. Chen, and Y. Zheng, ACS Nano 4(9), 5421 (2010).

26. V. V. Ginzburg and S. Balijepalli, Nano Lett. 7, 3716 (2007).

27. N. V. Svarovskaya, O. V. Bakina, E. A. Glazkova, M. I. Lerner, and S. G. Psakh'e, Russ. J. Phys. Chem. A 84, 1566 (2010).

28. S. Plimpton, J. Comput. Phys. 117, 1 (1995).

29. A. MacKerell, D. Bashford, M. Bellott, Dunbrack, et al., J. Phys. Chem. B 102, 3586 (1998).

30. H. Heinz, R. A. Vaia, B. L. Farmer, and R. R. Naik, J. Phys. Chem. C 112, 17281 (2008).

31. Hockney and Eastwood, Computer Simulation Using Particles (Adam Hilger, NY, 1989).

32. W. Humphrey, A. Dalke, and K. Schulten, J. Molec. Graphics 14.1, 33 (1996).

33. V. Sadovnichy, A. Tikhonravov, Vl. Voevodin, and V. Opanasenko, in Contemporary High Performance Computing: From Petascale toward Exascale (CRC Press, Boca Raton, USA, 2013), pp. 283-307. 